\title{
Forum
}

\section{Research, conservation and zoos: the EC Zoos Directive - a response to Rees}

\author{
Stephanie Wehnelt and Roger Wilkinson
}

Rees (2005) states in his critical review of the EC Zoos Directive that 'zoos have no incentive to undertake conservation research because they can legitimately ignore this requirement providing they carry out an alternative conservation measure. Zoos therefore can comply with the EU Zoos Directive by doing nothing.' Zoo conservation took a major step forward when the Council of EC Environment Ministers agreed in 1998 to an EC Zoos Directive to strengthen the conservation role of zoos. The Directive came into force in 1999 and requires that all Member States set up national systems for the licensing and inspection of zoos. The Zoo Licensing Act 1981 already implements many of the measures in the Directive, including the provision of proper accommodation and care for the animals, keeping up to date records, and taking appropriate measures to prevent escapes. But the requirements that zoos participate in conservation and education activities are new. Although many zoos already participate, the new legislation has made this a statutory requirement. Each European country is now responsible for enforcing the EU Directive with their national zoos. The Directive needs to be general because it applies to animal collections of very different sizes and structures. It cannot be expected that the Directive sets standards higher than is achievable for its smallest members.

Rees seems to misinterpret the emphasis of the Directive's aim, which is to further the conservation role of zoos, not to improve the quality of zoo research. Pure research in itself is not a legal requirement of the EC Zoos Directive, but an option to support a zoo's conservation role. If a zoo cannot carry out its own research, the Directive gives a list of alternative conservation related activities. Small zoos without the resources or staff expertise to conduct research can thus fulfil their conservation mission and meet legal requirements by facilitating research by others on site and by providing information to zoo researchers.

\footnotetext{
Stephanie Wehnelt Zoo Schmiding, Schmidingerstraße 5, A-4631

Krenglbach, Austria. E-mail s.wehnelt@zooschmiding.at

Roger Wilkinson Chester Zoo, North of England Zoological Society, Caughall Road, Upton, Chester, CH2 1LH, UK.

Received 8 December 2004. Accepted 24 January 2005.
}

Within the existing legislation an approach of proportionality is adopted. Small animal holdings (e.g. pet zoos) cannot be legally expected to make a major contribution to conservation research programmes but larger zoos will be criticised by zoo inspectors if their conservation and research actions are not appropriate for their size. The concept of proportionality is embedded in the Zoos Forum Handbook as well as in DEFRA's Zoo Licensing Act 1981 (Circular 02/2003, Conservation Measures for Zoos, page 5, bullet 9): 'The conditions must be appropriate to the size and nature of the zoos. For instance there is no expectation that small zoos should undertake major conservation and educational projects. The extent of a zoo's conservation and educational activities should be proportionate to its size and the diversity of its collection.' This approach is also embedded in the Zoos Forum Handbook. Although a small collection does not need to carry out research itself, Rees is incorrect in assuming that zoos can 'get away by doing nothing'. It is also important to point out that other means such as education are powerful tools of conservation. Small zoos that generate funds for in situ conservation programmes and also take their conservation education role seriously contribute substantially to conservation, even if they only indirectly contribute to research by, for example, permitting research, providing post mortem samples and by taking part in conservation breeding programmes. Therefore, the Directive, with all its accompanying documents and handbooks, is a useful document that sets reasonable standards. Zoos support and encourage cooperation with and active input from research academics and deserve support from them in working together on research that benefits conservation. All too often undirected academic research can be without direct value to conservation.

Rees defines research as 'systematic collection and analysis of data or the development of new techniques'. However, research activities are not all so highbrow. By participating in coordinated breeding programmes a collection contributes to research by providing information about their animals, which studbook keepers can analyse. Additionally, zoos of all sizes encourage academics and zoo funded researchers to study their animals. Many provide post mortem samples to research institutions and museums. These contributions are valuable additions to the research roles of zoos and should be included in the definition of zoo research. The strength of 
zoos lies in offering an entirely unique research facility. This can be maximised by scientifically trained zoo staff maintaining strong and long-term links with academic and conservation organizations.

Rees' view that the main conservation role of zoos is to contribute to reintroduction programmes is somewhat dated. Zoos have moved on from seeing their only conservation role as reintroducing captive animals into their natural habitat. Research driven by IUCN, zoos and other conservation organizations has concluded that relocation and restocking programmes of wild animals may be more successful to help species survive in situ, and many zoos take an active, leading part in such programmes. Ex situ conservation activities of zoos mainly include conservation education and managing safety net populations of species listed on the IUCN Red List (IUCN, 2004). More recently zoos have also engaged in research in long-term preventative conservation activities such as artificial insemination techniques, and cryobiology of sperm, eggs, tissue, and entire organisms, all of which may play a crucial role in the future conservation of threatened species.

With regards to the research output of zoos, Rees confuses research projects conducted for academic training purposes with higher level research driven by zoos, conservationists and Taxon Advisory Groups to further conservation (i.e. breeding, husbandry, welfare, in situ conservation, education). Zoos collaborate with many other conservation and academic organizations in stimulating, funding, collaborating on and developing research programmes. The list of projects used by Semple (2002) includes mainly undergraduate student projects, which represent the large number of projects where zoos are utilized by academic institutions for training. In many zoos only non-invasive and non-manipulative research is permitted on ethical grounds, and as such the majority of research projects are observational, as reflected in Rees' findings on publications in Zoo Biology. Zoos offer a unique training venue for future zoologists and wildlife veterinarians that should not be undervalued, even if the direct benefits of academic training projects are unclear and the nature of such projects is driven by academic rather than applied needs. At Chester Zoo, for example, over 150 university students benefit from such training opportunities each year.
Rees criticizes zoo research as being 'conducted in unnatural conditions and often with very small samples of animals'. Research should be carried out with the species and in the location best suited to answer the scientific question, whether in the natural habitat or in a zoo or aquarium. Of course most research on wild animals should preferably be carried out in their natural habitat. However, if this is not possible because there are insufficient individuals remaining in the wild, or because the species in question is secretive or nocturnal or otherwise difficult to access, then studying these animals in zoos is often the only alternative, and in many cases even the better alternative, to studies in the wild. Benefits of research in zoos are that the animals are individually identifiable, are used to the presence of observers, and the environment can be controlled to some extent. Many field studies use a restricted number of animals, often from the same population, and field researchers often struggle with the identification and following of individuals. Small sample sizes do not automatically mean that scientific investigations are invalid. Recently developed statistical methods such as randomization tests allow the analysis of small samples. As long as the constraints of the validity of the results for the rest of the population are highlighted, answers applicable to the studied individuals can be made on valid, statistical grounds.

Those who attend national and international zoo research and conservation meetings will be aware of the wealth of research involvement of zoos and of the recent developments within zoo conservation and research. Rees' criticisms of zoo conservation research highlight the necessity for publicizing more widely the achievements of modern zoos in conservation and research and building more bridges with the academic community.

\section{References}

IUCN (2004) IUCN Red List of Threatened Species. IUCN, Gland, Switzerland [http:/ / www.redlist.org, accessed 24 January 2005].

Rees, P.A. (2005) Will the EC Zoos Directive increase the conservation value of zoo research? Oryx, 39, 128-131.

Semple, S. (2002) Analysis of research projects conducted in Federation collections to 2000. Federation Research Newsletter, 3, 3. Federation of Zoological Gardens of Great Britain and Ireland. 